\title{
Magnetic ordering above room temperature in the sigma-phase of $\mathrm{Fe}_{66} \mathrm{~V}_{34}$
}

\author{
Jakub Cieślak ${ }^{\mathrm{a}}$, Benilde F.O. Costa ${ }^{\mathrm{b}}$, Stanisław M. Dubiel ${ }^{\mathrm{a}, *}$, Michael Reissner ${ }^{\mathrm{c}}$, Walter Steiner ${ }^{\mathrm{c}}$ \\ a Faculty of Physics and Computer Science, AGH University of Science and Technology, 30-059 Kraków, Poland \\ ${ }^{\mathrm{b}}$ CEMDRX Department of Physics, University of Coimbra, 3000-516 Coimbra, Portugal \\ c Institute of Solid State Physics, Vienna University of Technology, 1040 Wien, Austria
}

\section{A R T I C L E I N F O}

\section{Article history:}

Received 22 October 2008

Received in revised form

2 December 2008

PACS:

75.30.Cr

75.50.Bb

$76.80 .+\mathrm{y}$

77.80.Bh

Keywords:

Sigma-phase

Fe-V system

Magnetic property

Curie temperature

\begin{abstract}
A B S T R A C T
Magnetic properties of four sigma-phase $\mathrm{Fe}_{100-x} \mathrm{~V}_{x}$ samples with $34.4 \leqslant x \leqslant 55.1$ were investigated by Mössbauer spectroscopy and magnetic measurements in the temperature interval 4.2-300 K. Four magnetic quantities, viz. hyperfine field, Curie temperature, magnetic moment and susceptibility, were determined. The sample containing 34.4 at\% $\mathrm{V}$ was revealed to exhibit the largest values found up to now for the sigma-phase for average hyperfine field, $\langle B\rangle=12.1 \mathrm{~T}$, average magnetic moment per Fe atom, $\langle\mu\rangle=0.89 \mu_{\mathrm{B}}$, and Curie temperature, $T_{C}=315.3 \mathrm{~K}$. The quantities were shown to be strongly correlated with each other. In particular, $T_{C}$ is linearly correlated with $\langle\mu\rangle$ with a slope of $406.5 \mathrm{~K} / \mu_{\mathrm{B}}$, as well as $\langle B\rangle$ is so correlated with $\langle\mu\rangle$, yielding $14.3 \mathrm{~T} / \mu_{\mathrm{B}}$ for the hyperfine coupling constant.
\end{abstract}

(c) 2009 Elsevier B.V. All rights reserved.

\section{Introduction}

Among over 50 examples of the $\sigma$-phases (tetragonal unit cellstructure type $\mathrm{D}_{4 \mathrm{~h}}^{14} \mathrm{P}_{2} / \mathrm{mnm}$ ) known to exist in binary alloy systems, only two, viz. $\sigma-\mathrm{FeCr}$ and $\sigma-\mathrm{FeV}$, exhibit magnetic order [1]. Magnetism of the $\sigma$-phase in the $\mathrm{Fe}-\mathrm{Cr}$ system can be termed as weak itinerant, with the highest Curie temperature $T_{C}=39 \mathrm{~K}$, and the largest average magnetic moment per Fe atom $\langle\mu\rangle=0.29 \mu_{\mathrm{B}}$ found in the sample with 45 at\% $\mathrm{Cr}$ [2]. Exchange interaction in the $\sigma$-phase of the Fe- $\mathrm{V}$ system is much stronger. Here, the highest value of $T_{C}=267.5 \mathrm{~K}$ was recorded for a sample with 37.3 at\% V [3], and the largest value of $\langle\mu\rangle=0.92 \mu_{\mathrm{B}}$ was reported for a sample containing 36 at\% $\mathrm{V}$ [4]. However, the latter was not directly measured, but estimated from the average hyperfine field using a conversion factor (hyperfine coupling constant) of $14.7 \mathrm{~T} / \mu_{\mathrm{B}}$. The largest value of $0.62 \mu_{\mathrm{B}}$ was derived from magnetization measurements for a $\sigma-\mathrm{Fe}_{63.8} \mathrm{~V}_{36.2}$ sample [5].

It should be noted that the difference in the values of the above-mentioned magnetic quantities, i.e. $T_{C}$ and $\langle\mu\rangle$ found for the $\sigma$-phase in the $\mathrm{Fe}-\mathrm{Cr}$ and $\mathrm{Fe}-\mathrm{V}$ systems, does not only originate from the difference in a composition at which these extreme values were measured. In fact, if one makes such

\footnotetext{
* Corresponding author.

E-mail address: dubiel@novell.ftj.agh.edu.pl (S.M. Dubiel).
}

comparison of the magnetic quantities for similar compositions, one finds a significant difference for the two systems. In particular, the difference in the magnetic moment found for the two systems increases linearly with the increase in $\mathrm{Fe}$ content in favour of $\sigma-\mathrm{FeV}$, i.e. the rate of increase is higher for $\sigma-\mathrm{FeV}$ than the one for $\sigma-\mathrm{FeCr}[6,7]$.

The present investigation was motivated by our previous results recorded on a series of $\sigma-\mathrm{Fe}_{100-x} \mathrm{Cr}_{x}$ samples with $45 \leqslant x \leqslant 50$. The results were obtained using magnetization measurements and Mössbauer-effect techniques [2,8].

First, we found that the values of $\langle\mu\rangle$ determined from magnetization measured in external magnetic fields, $B_{a}$, up to $15 \mathrm{~T}$ were shifted by $\sim 0.1 \mu_{\mathrm{B}}$ to higher values in comparison with their counterparts derived from similar curves recorded in an external field of $1 \mathrm{~T}[6,7]$. This difference follows from the ill-defined extrapolation condition of the magnetization measured in the external magnetic field of $1 \mathrm{~T}$ to $B_{a}=0 \mathrm{~T}$.

Second, we have revealed that the correlation of the average hyperfine field with $\langle\mu\rangle$ is not linear. In other words, the conversion factor (hyperfine coupling constant) changes continuously its value with composition between $\sim 9 \mathrm{~T} / \mu_{\mathrm{B}}$ for $x$ close to 45 and $\sim 18 \mathrm{~T} / \mu_{\mathrm{B}}$ for $x$ close to $50[8]$.

In the light of the above given information, it is of interest to verify whether or not similar effects can be found for the $\sigma-\mathrm{FeV}$ phase, which is in fact more appropriate for testing the abovementioned characteristics because the composition range of the 
$\sigma$-phase occurrence in $\mathrm{Fe}-\mathrm{V}$ is by a factor $\sim 6$ larger than the one in the $\mathrm{Fe}-\mathrm{Cr}$ system [9].

\section{Sample preparation}

Master alloys of $\alpha-\mathrm{Fe}_{100-x} \mathrm{~V}_{x}$ with a nominal composition $x=36,40,48$ and 60 were prepared by melting appropriate amounts of $\mathrm{Fe}(99.95 \%$ purity) and $\mathrm{V}$ (99.5\% purity) in an arc furnace under protective argon atmosphere. The ingots received after melting were next solution treated at $1273 \mathrm{~K}$ for $72 \mathrm{~h}$ followed by a water quench. The chemical composition was determined on the homogenized samples by electron probe microanalysis as $x=34.4,39.9,47.9$ and 55.1. The transformation into the $\sigma$-phase was performed by annealing the ingots at $T_{a}=973 \mathrm{~K}$ for 25 days. The verification of the $\alpha$ to $\sigma$ phase transformation was done by recording room-temperature $\mathrm{X}$-ray and neutron diffraction patterns [10]. It should be noted that the $\sigma$-phase samples contained some small fraction of $\mathrm{V}$-rich precipitates, most likely in the form of carbides. These precipitates were not detected in the neutron diffraction patterns. However, it is known that carbide nucleation often precedes $\sigma$-phase nucleation [11].

\section{Results and discussion}

\subsection{Curie temperature}

\subsubsection{Mössbauer-effect measurements}

${ }^{57} \mathrm{Fe}$ Mössbauer spectra were recorded in the temperature interval of $4.2-300 \mathrm{~K}$ with a standard spectrometer and a ${ }^{57} \mathrm{Co} / \mathrm{Rh}$ source of $\gamma$-rays. The temperature of a sample, which was placed in a cryostat, was kept constant to within $\pm 0.2 \mathrm{~K}$. The shape of the spectra sensitively depends on sample composition and, for a given composition, on temperature. To illustrate the former, a set of the spectra recorded at $4.2 \mathrm{~K}$ for $x=34.4,39.9,47.9$ and 55.1 is presented in Fig. 1. The influence of temperature on the shape of the spectrum is shown in Fig. 2 for $x=34.4$.

The spectra, because of their shape and the underlying crystallographic structure (five different crystallographic sites with high (12-15) coordination number and chemically disordered distribution of Fe and $\mathrm{V}$ atoms over these sites), were fitted with the hyperfine field distribution (HFD) method to get the distribution of the hyperfine field, $P(B)$. It was assumed that the hyperfine field was linearly correlated with the isomer shift and the quadrupole splitting. The former assumption is justified both by experimentally found evidence for such correlation in binary bcc-alloys of iron alloys including Fe-V [12] and on the recent theoretical calculation of the electronic structure for a $\sigma-\mathrm{FeCr}$ compound in a paramagnetic state [13]. According to the latter, changes in the electronic structure due to $\mathrm{Cr}$ atoms are in line with the corresponding changes in the $\alpha$-FeCr alloys. There is weak evidence concerning the correlation between the hyperfine field and the quadrupole splitting, though there is also some experimental data in favour of it $[14,15]$. In addition, the fitting procedure was carried out assuming the ratio of the lines within a sextet was 3:2:1 and that all line widths were the same. Some examples of the $P(B)$-curves obtained in this way are presented in Fig. 3. By their integration, the average hyperfine field, $\langle B\rangle$, was calculated. From the temperature dependence of $\langle B\rangle$, the Curie temperature, $T_{C 1}$, was estimated for each sample. In particular, from the $\langle B\rangle(T)$ plot obtained for the $\sigma-\mathrm{Fe}_{65.6} \mathrm{~V}_{34.4}$ sample, which is shown in Fig. $4, T_{C}=324 \mathrm{~K}$ was derived (the non-zero value of $\langle B\rangle$ at $T \geqslant 324 \mathrm{~K}$ follows from the fact that the spectrum in the paramagnetic phase is not a single line, because there are five different crystallographic sites occupied by Fe (and V atoms), so the isomer shift is different for each site; in addition, their
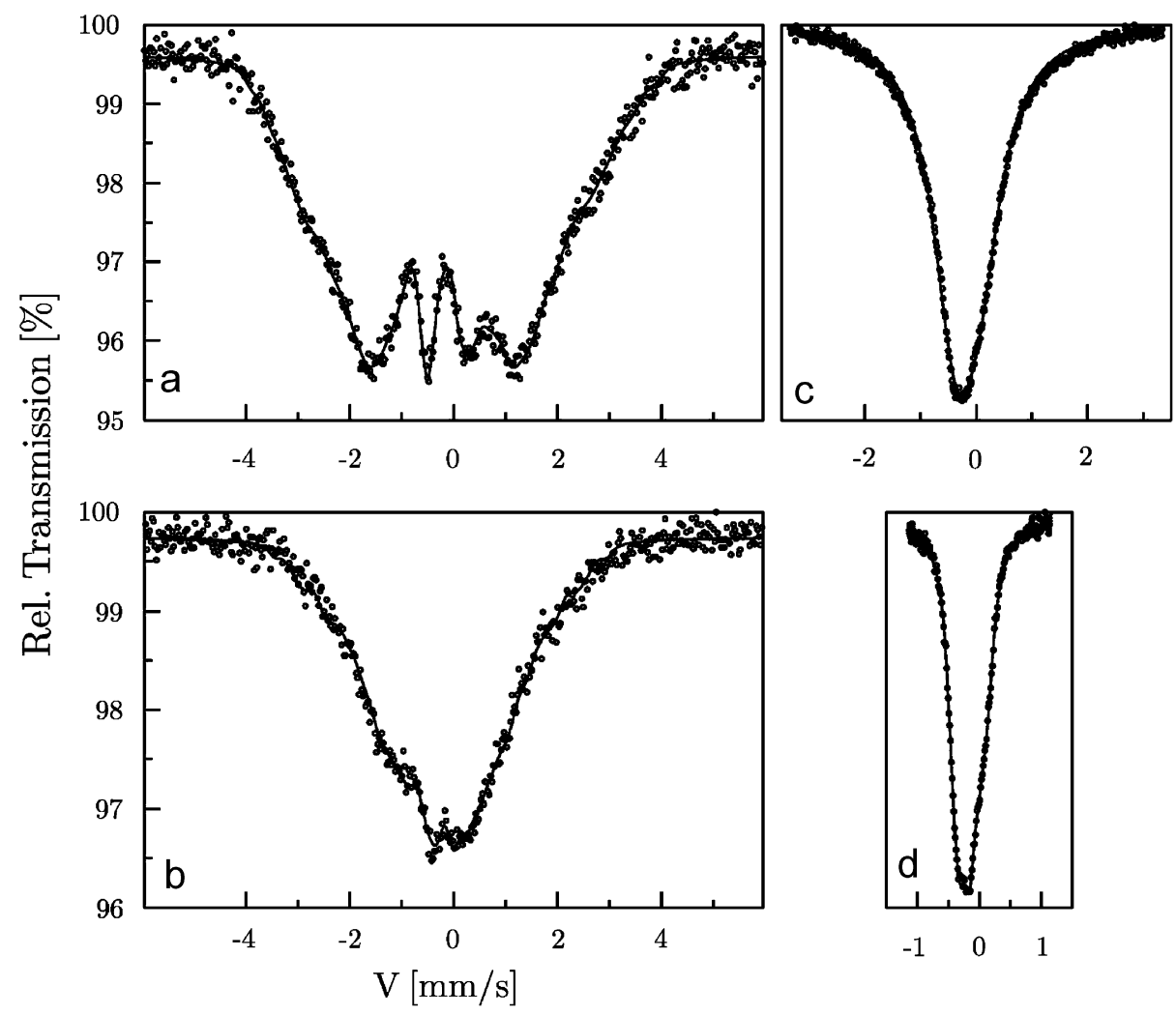

Fig. 1. ${ }^{57} \mathrm{Fe}$ Mössbauer spectra recorded at $4.2 \mathrm{~K}$ on the $\sigma-\mathrm{Fe}_{100-x} \mathrm{~V}_{x}$ samples: (a) $x=34.4$, (b) $x=39.9$, (c) $x=47.9$ and (d) $x=55.1$. 


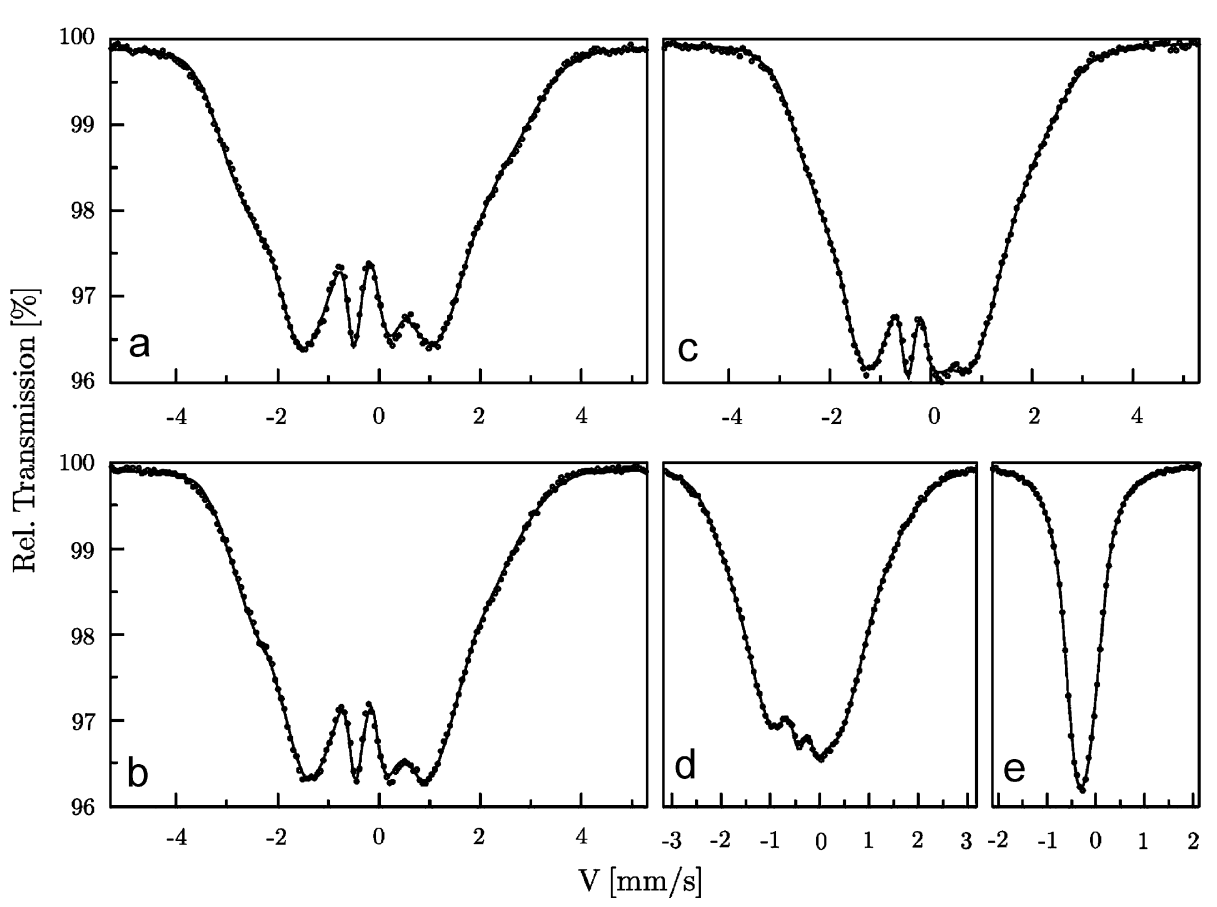

Fig. 2. ${ }^{57} \mathrm{Fe}$ Mössbauer spectra recorded on the $\sigma-\mathrm{Fe}_{65.6} \mathrm{~V}_{34.4}$ sample at: (a) $4.2 \mathrm{~K}$, (b) $120 \mathrm{~K}$, (c) $195 \mathrm{~K}$, (d) $269 \mathrm{~K}$ and (e) $330 \mathrm{~K}$.
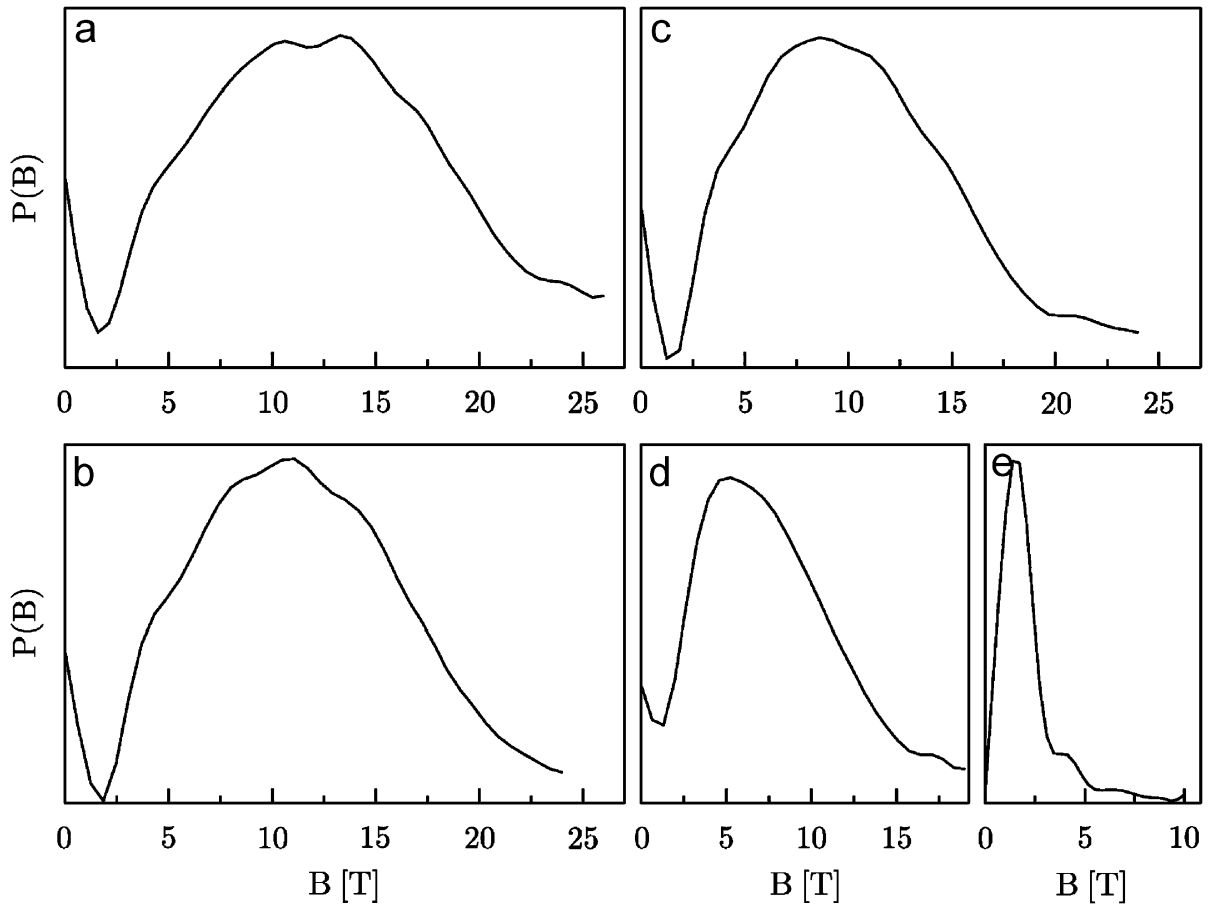

Fig. 3. Distribution of the hyperfine field for the $\sigma-\mathrm{Fe}_{65.6} \mathrm{~V}_{34.4}$ sample obtained from the spectra recorded at: (a) $4.2 \mathrm{~K}$, (b) $120 \mathrm{~K}$, (c) $195 \mathrm{~K}$, (d) $269 \mathrm{~K}$ and (e) $330 \mathrm{~K}$.

symmetry is lower than cubic, so each subspectrum can be quadrupole split. As it was fitted with the HFD method, the departure from the single line was accounted for by a small hyperfine field). To our best knowledge, this is the highest value of Curie temperature ever recorded for a $\sigma-\mathrm{FeV}$ sample.

\subsubsection{Magnetization versus temperature measurements}

Measurements of magnetization, $M$, were performed with a vibrating sample magnetometer (VSM) in a constant magnetic field, $B_{a}$, versus temperature, $T$. Typical curves are shown in the upper part of Fig. 5. By plotting $\mathrm{d} M / \mathrm{d} T$ versus $T$-see lower part of Fig. 5-the Curie points were derived. In particular, the value of $T_{C 2}=306.6 \mathrm{~K}$ was found for the $\sigma-\mathrm{Fe}_{65.6} \mathrm{~V}_{34.4}$ sample. It is smaller than the corresponding value evaluated from the average hyperfine field, but it is known that such difference between the two methods may occur as far as the Curie point is concerned due to (a) different time-scale and (b) spin correlation lengths involved in these two techniques. In any case, the record highvalue of $T_{C 1}$ found from the $\langle B\rangle(T)$ plot has been confirmed with 


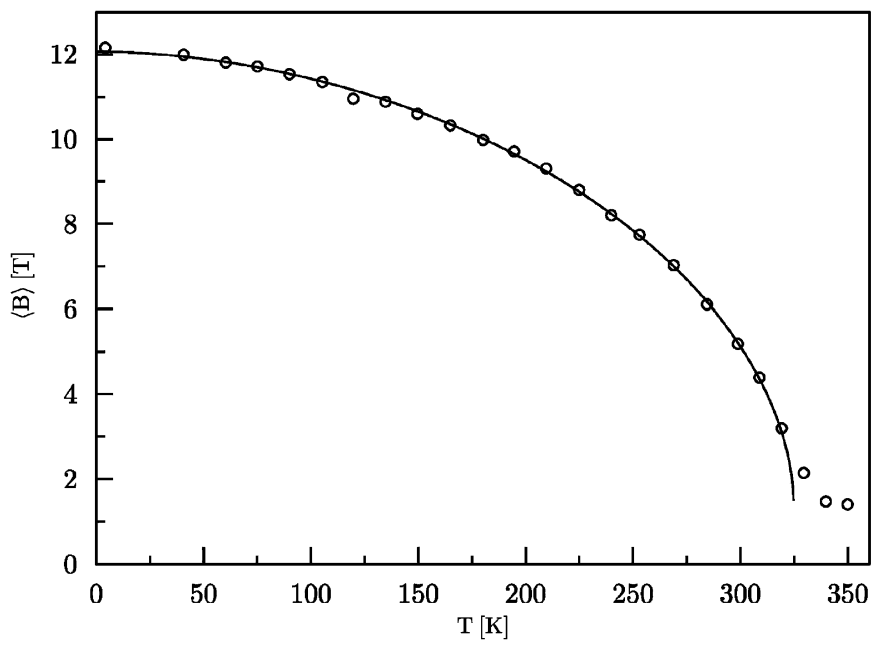

Fig. 4. Average hyperfine field, $\langle B\rangle$, versus temperature, $T$, for the $\sigma-\mathrm{Fe}_{65.6} \mathrm{~V}_{34.4}$ sample with the estimated value of the Curie point $T_{C}=324 \mathrm{~K}$.

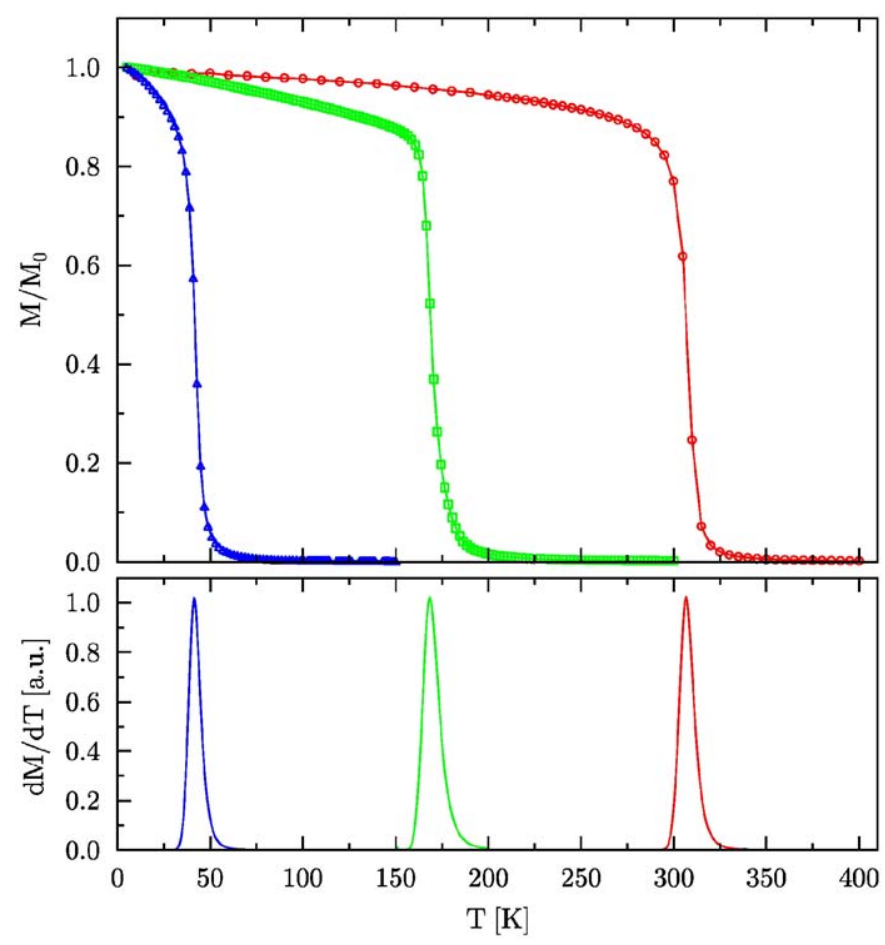

Fig. 5. Normalized magnetization curves, $M / M_{o}$ ( $M_{o}$ being the value of $M$ at $4.2 \mathrm{~K}$ and $B_{a}=100,700$ and 1760 e, respectively) versus temperature $T$ (top) and $\mathrm{d} M / \mathrm{d} T$ curves (bottom) for three of the studied samples $(x=34.4,39.9$ and 47.9 in sequence from right to left)

the $M(T)$ plot. $T_{C}$-values being the arithmetic average over $T_{C 1}$ and $T_{C 2}$ are presented in Fig. 6, together with other values obtained in this study, as well as those found in the literature [3,5-7,16-19]. A non-linear dependence on compound composition can be readily seen.

\subsection{Magnetic moment and susceptibility}

Magnetic moment was derived from the magnetization measurements in an external magnetic field $B_{a} \leqslant 9 \mathrm{~T}$ at constant temperature. Typical $M\left(B_{a}\right)$-curves are presented in Fig. 7. By extrapolation of the linear part of the $M\left(B_{a}\right)$-curve recorded at $5 \mathrm{~K}$

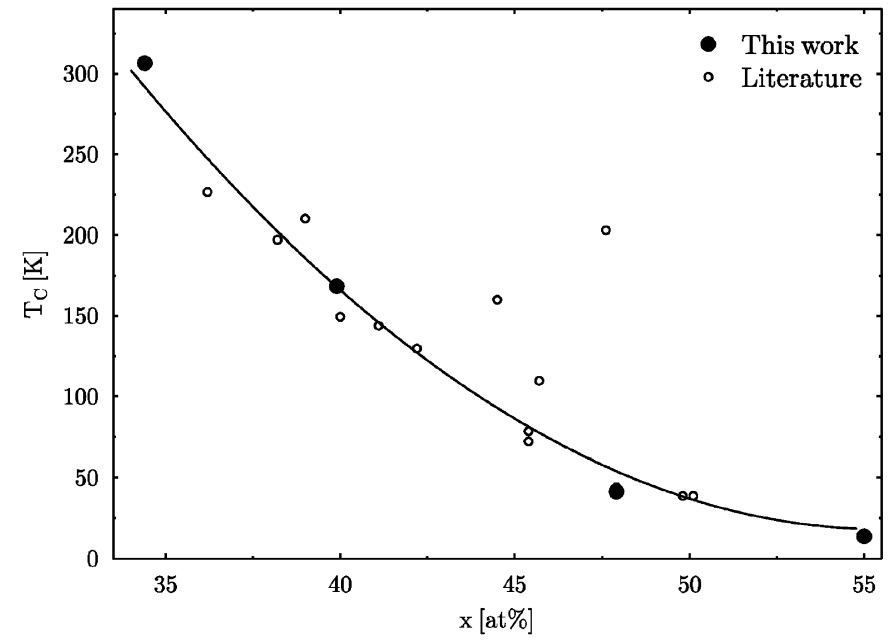

Fig. 6. Curie temperature, $T_{C}$, versus $\mathrm{V}$ content, $x$ for the $\sigma-\mathrm{FeV}$ compounds. The solid line is a guide to the eye. Empty symbols are taken from the literature [3,5-7,12-15].

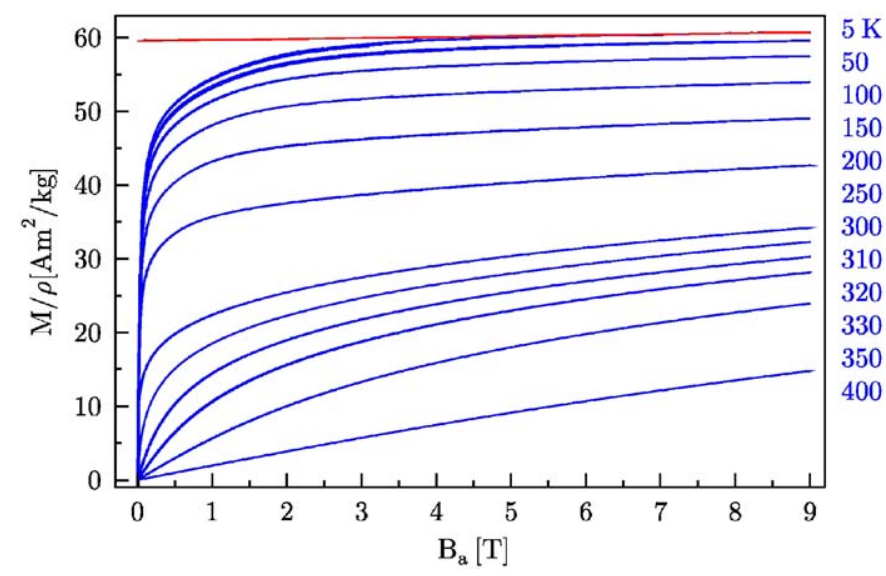

Fig. 7. Magnetization curves recorded on the $\sigma-\mathrm{Fe}_{65.6} \mathrm{~V}_{34.4}$ sample versus external magnetic field, $B_{a}$, for various temperatures shown. Saturation magnetization was determined by extrapolation of the linear part of the curve measured at $5 \mathrm{~K}$ to $B_{a}=0 \mathrm{~T}$ as indicated.

to $B_{a}=0 \mathrm{~T}$, the saturation magnetization value $M_{s}$ was found, and from it the average magnetic moment per Fe atom, $\langle\mu\rangle$, was evaluated. Its non-linear dependence on the compound composition can be seen in Fig. 8, where both the values found in this study as well as those known from the literature are plotted. An upward shift of the presently found data relative to those from the literature can be readily seen. The reason for the shift, also revealed previously for $\sigma$ - $\mathrm{FeCr}$ samples [2], is a result of the extrapolation condition of the magnetization curves to $B_{a}=0 \mathrm{~T}$. Our extrapolation procedure was from much larger $B_{a}$-values, hence more reliable. This, in the light of a non-saturating character of the $M\left(B_{a}\right)$-curves, had resulted in different zero-field saturation values, hence $\langle\mu\rangle$. The $M\left(B_{a}\right)$ data recorded for $x=39.9$ and 47.9 at $T>T_{C 2}$ were used to determine the effective paramagnetic moment, $\mu_{\text {eff, }}$ which was next used to verify the Rhodes-Wohlfarth criterion for itinerant magnetism. For this purpose the susceptibility, $\chi=M / B_{a}$, was determined. An example of the data is displayed in Fig. 9. The linear part of the inverse susceptibility was fitted to the Curie-Weiss formula:

$\chi=\chi_{o}+\frac{C}{T-\Theta}$ 


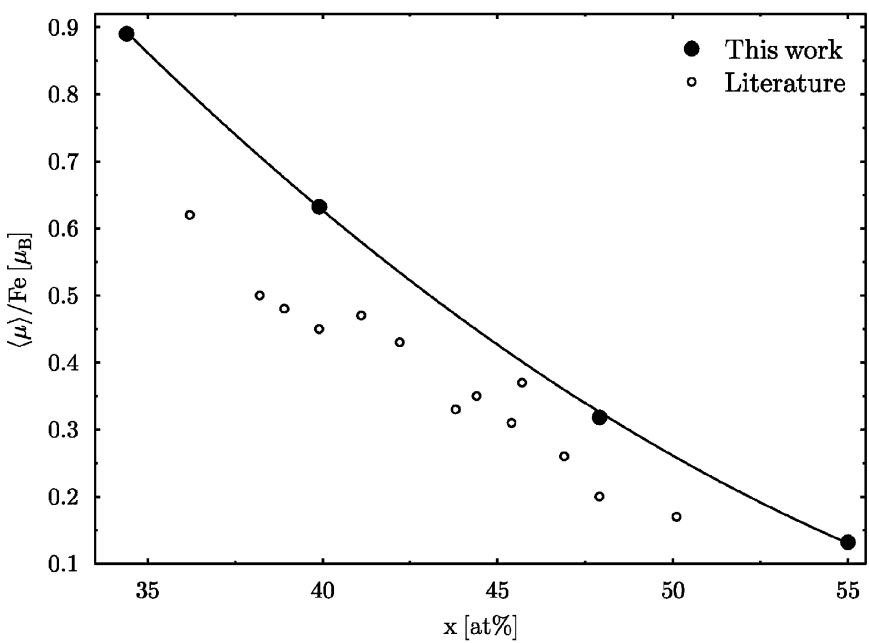

Fig. 8. Magnetic moment per Fe atom, $\langle\mu\rangle$, for the $\sigma-\mathrm{FeV}$ compounds versus $\mathrm{V}$ content, $x$. The solid line is to guide the eye. Empty symbols represent data taken from the literature $[3,5-7,12-15]$.

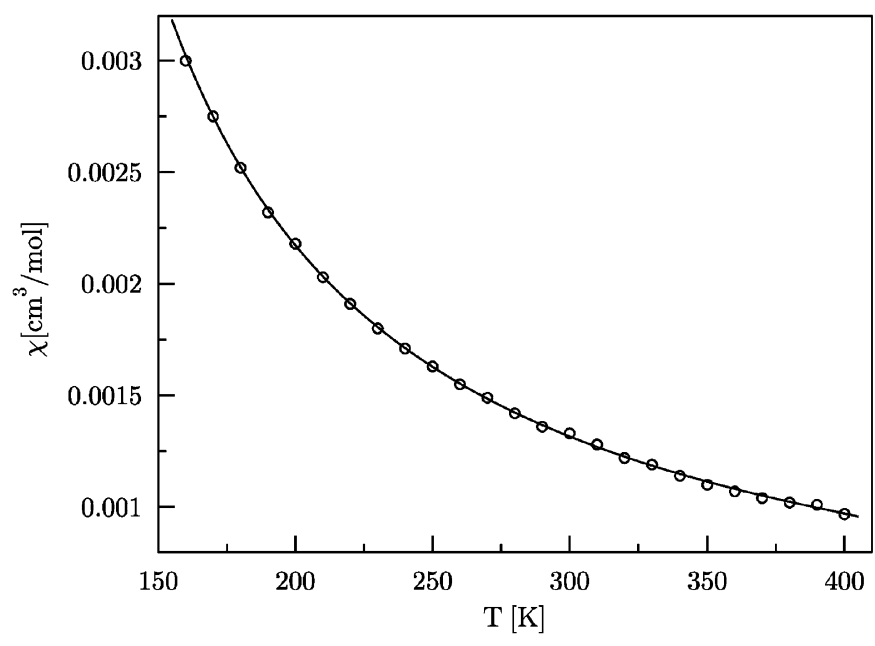

Fig. 9. Susceptibility versus temperature for the $\sigma-\mathrm{Fe}_{66.6} \mathrm{~V}_{34.4}$ sample. The solid line is the best-fit to the data in terms of equation (1).

Table 1

Magnetic quantities determined for the studied $\sigma-\mathrm{Fe}_{100-x} \mathrm{~V}_{x}$ samples.

\begin{tabular}{lrrrlllr}
\hline$x$ (at\%) & $T_{\mathrm{C} 1}(\mathrm{~K})$ & $T_{\mathrm{C} 2}(\mathrm{~K})$ & $\langle B\rangle(\mathrm{T})$ & $\langle\mu\rangle\left(\mu_{\mathrm{B}}\right)$ & $\mu_{\text {eff }}\left(\mu_{\mathrm{B}}\right)$ & $\mu_{\mathrm{s}}\left(\mu_{\mathrm{B}}\right)$ & $\Theta(\mathrm{K})$ \\
\hline 34.4 & 324.0 & 306.6 & 12.2 & 0.89 & - & 0.58 & - \\
39.9 & 149.0 & 168.4 & 8.8 & 0.63 & 2.162 & 0.38 & 237 \\
47.9 & 40.0 & 41.4 & 4.4 & 0.32 & 2.005 & 0.165 & 68 \\
55.1 & 17.0 & 13.8 & 2.1 & 0.13 & - & 0.06 & - \\
\hline
\end{tabular}

The Curie temperature as determined from the Mössbauer measurements is denoted by $T_{C 1}$, that from the magnetization measurements by $T_{C 2}$, the average hyperfine field by $\langle B\rangle$, the average magnetic moment per Fe atom by $\langle\mu\rangle$, the magnetic saturation moment, $\mu_{\mathrm{s}}$, the effective paramagnetic moment by $\mu_{\text {eff }}$ and the paramagnetic Curie temperature by $\Theta$.

The best-fit values of the parameters obtained in such a way are displayed in Table 1. From $C$ the $\mu_{\text {eff }}$-values were derived. The Curie temperature, $T_{C}$, the average magnetic moment per Fe atom, $\langle\mu\rangle$, and the average hyperfine field, $\langle B\rangle$, are also included in this table. From the data, and in particular from the $\mu_{\text {eff }} / \mu_{s}$ ratio, $\mu_{s}$ being the saturation moment, it is clear that according to the Rhodes-Wohlfarth criterion, the magnetism of the studied system has itinerant character.

\subsection{Curie point-magnetic moment correlation}

It is well known that magnetizations as a function of the valence electron number per atom of $3 \mathrm{~d}$ transition metal systems form the so-called Slater-Pauling curve. Similarly, the Curie temperatures of these alloys also exhibit a Slater-Pauling-like behaviour [20].

Though the overall shape of the latter is like the former, the two differ in details. It follows from this fact that the Curie temperature depend not only on the magnetization but also on the magnetic exchange interactions coefficients that are characteristic of a given alloy system.

Indeed, recent theoretical calculations carried out with the KKR-CPA-LDA method for several 3d transition binary alloys have clearly demonstrated that the Curie temperature-magnetic moment relationship is characteristic of a given system [21]. In other words, the two quantities are, in general, not linearly correlated.

As shown in Fig. 10 for the $\sigma-\mathrm{FeV}$ system, $T_{C}$ is very well linearly correlated with the magnetic moment, $\langle\mu\rangle$. The slope is equal to $406.5 \mathrm{~K} / \mu_{\mathrm{B}}$, and is significantly different from the one found recently for the $\sigma$-phase in the $\mathrm{Fe}-\mathrm{Cr}$ system (i.e. $205 \mathrm{~K} / \mu_{\mathrm{B}}$ ) $[2,8]$. A linear correlation between the two magnetic quantities was also observed in the $\alpha$-phase of the Fe-V system of similar composition as the one in the presently studied samples [4]. The slope for the latter is, however, equal to $741 \mathrm{~K} / \mu_{\mathrm{B}}$, i.e. almost twice the one in the $\sigma$-FeV system.

For comparison, the $T_{C} / \mu$ value for a pure iron is equal to $470 \mathrm{~K} /$ $\mu_{\mathrm{B}}$. This comparison clearly shows that the $T_{C}-\langle\mu\rangle$ relationship depends not only on the alloy system but, for a given system, also on its crystallographic structure.

\subsection{Average hyperfine field-magnetic moment correlation}

As illustrated in Fig. 11 for the $\sigma-\mathrm{FeV}$ compounds, the average hyperfine field, $\langle B\rangle$, is linearly correlated with the average magnetic moment per Fe atom, $\langle\mu\rangle$, with the slope of $14.3 \mathrm{~T} / \mu_{\mathrm{B}}$. Such correlation is rather strange and unexpected for this system as according to theoretical calculations performed on bcc-Fe systems [22-24], only a part of the hyperfine field, viz. the one due to a polarization of the core electrons, is proportional to the Fesite magnetic moment, while the second part, viz. the one due to the polarization of the conduction electrons, $B_{C E P}$, is not. In other

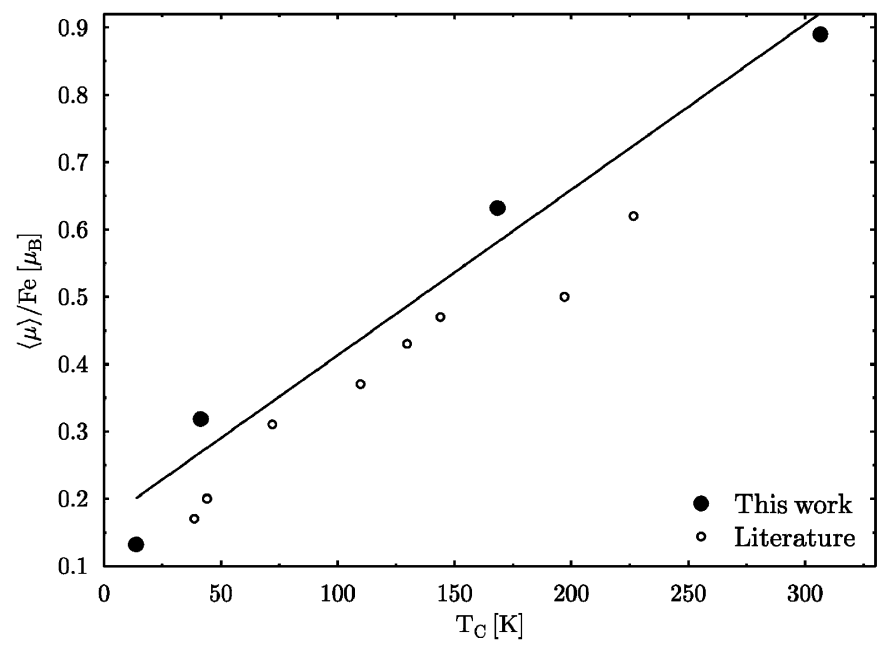

Fig. 10. Linear correlation between the average magnetic moment per Fe atom, < $\mu>$, and the Curie temperature, $T_{C}$, for the $\sigma-\mathrm{FeV}$ compounds. The solid line is the best-fit to the data. The extreme points span over the whole concentration range of the $\sigma$-phase existence. 


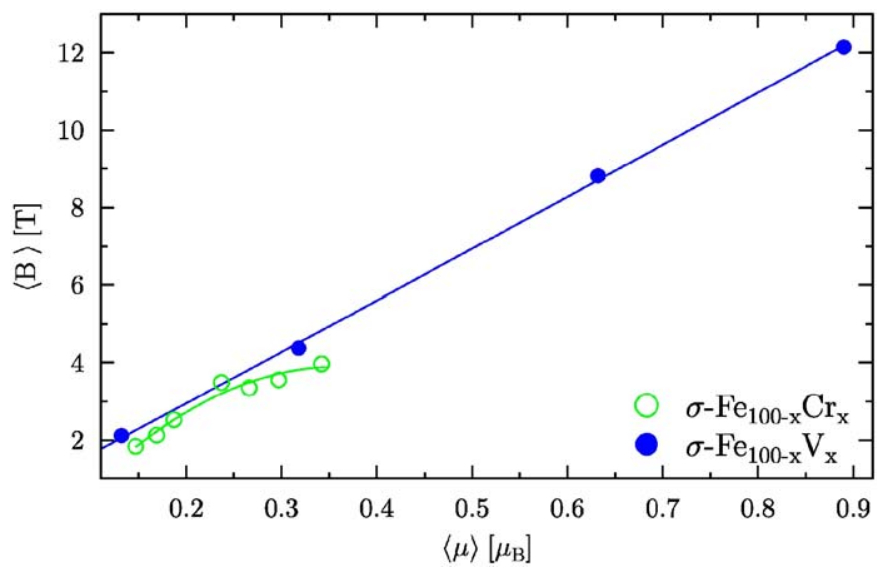

Fig. 11. Average hyperfine field, $\langle B\rangle$, versus average magnetic moment per Fe atom, $\langle\mu\rangle$, in the $\sigma$-phase in the $\mathrm{Fe}-\mathrm{Cr}[8]$ and $\mathrm{Fe}-\mathrm{V}$ systems. The solid lines are the guide to the eye.

words, a non-linear $\langle B\rangle-\langle\mu\rangle$ relationship might be taken as evidence that a substantial contribution to the hyperfine field originates from the conduction electrons, i.e. the system is magnetically itinerant. In the presently studied case, the Rhodes-Wohlfarth criterion speaks in favour of the latter. The linear $\langle B\rangle-\langle\mu\rangle$ relationship means that also the $B_{C E P}$ term is proportional to $\langle\mu\rangle$. This conclusion must be, however, verified by theoretical calculations devoted to the investigated system.

The presently found correlation between $\langle B\rangle$ and $\langle\mu\rangle$ is also of a practical meaning as it can be used as the reference plot permitting a unique transformation between the two quantities. It is linear and different than the one found previously for the $\sigma$-Fe- $\mathrm{Cr}$ system [8]. The latter feature gives clear evidence that the $\langle B\rangle-\langle\mu\rangle$ relationship is not universal, but it is rather characteristic of a given structure and system.

\section{Conclusions}

The results obtained in this study permit drawing the following conclusions:

(a) The $\sigma-\mathrm{Fe}_{65.6} \mathrm{~V}_{34.4}$ sample shows the strongest magnetic properties ever found for the sigma-phase as measured in terms of the Curie temperature, magnetic moment and magnetic hyperfine field.

(b) Curie temperature and magnetic moment per Fe atom show non-linear decrease with $\mathrm{V}$ content.

(c) Curie temperature and the average hyperfine field are linearly correlated with the magnetic moment. The latter is rather unexpected for an itinerant system and prompts theoretical calculations.

\section{Acknowledgement}

Dr. Jan Żukrowski is thanked for melting the samples.

\section{References}

[1] E.O. Hall, S.H. Algie, Metall. Rev. 11 (1966) 61.

[2] J. Cieslak, M. Reissner, W. Steiner, S.M. Dubiel, J. Magn. Magn. Mater. 272-276 (2004) 534;

J. Cieslak, M. Reissner, W. Steiner, S.M. Dubiel, Phys. Status Solidi. 205 (2008) 1794.

[3] H.H. Ettwig, W. Pepperhoff, Arch. Eisenhuttenwes. 43 (1972) 271.

[4] A.M. van der Kraan, D.B. de Mooij, K.H.J. Buschow, Phys. Status Solidi (a) 88 (1985) 231.

[5] Y. Sumimoto, T. Moriya, H. Ino, F.E. Fujita, J. Phys. Soc. Japan 35 (1973) 461.

[6] D.A. Read, E.H. Thomas, IEEE Trans. Magn. MAG-2 (1966) 415.

[7] D.A. Read, E.H. Thomas, J.B. Forsythe, J. Phys. Chem. Solids 29 (1968) 1569.

[8] J. Cieślak, B.F.O. Costa, S.M. Dubiel, M. Reissner, W. Steiner, J. Phys.: Condens. Matter 17 (2005) 2985.

[9] O. Kubaschewski, Iron-Binary Phase Diagrams, Springer, Berlin, 1982.

[10] J. Cieślak, M. Reissner, S.M. Dubiel, J. Wernisch, W. Steiner, J. Alloys Compds. 20 (2008) 20.

[11] R. Blower, G.J. Cox, J. Iron Steel Inst. (1970) 769.

[12] S.M. Dubiel, W. Zinn, J. Magn. Magn. Mater. 45 (1984) 298.

[13] J. Cieślak, J. Toboła, S.M. Dubiel, S. Kaprzyk, W. Steiner, M. Reissner, J. Phys.: Condens. Matter 20 (2008) 235234.

[14] D.V. Parwate, A.N. Garg, J. Radioanal. Nucl. Chem. Lett. 87 (1984) 379

[15] I. Vincze, F. Van Der Woude, J.M. Friedt, Phys. Rev. B 33 (1986) 5050.

[16] D. Parsons, Nature 185 (1960) 839.

[17] M.V. Nevitt, P.A. Beck, Trans. AIME (1955) 669

[18] M. Mori, T. Mitsui, J. Phys. Soc. Japan 22 (1967) 931.

[19] M.V. Nevitt, A.T. Aldred, J. Appl. Phys. 34 (1963) 463.

[20] H.P. Wijn, in: R. Poerschke (Ed.), Magnetic Properties of Metals, Springer Berlin, 1991.

[21] C. Takahashi, M. Ogura, H. Akai, J. Phys.: Condens. Matter 19 (2007) 365233.

[22] R.E. Watson, A.J. Freeman, Phys. Rev. 123 (1961) 2027.

[23] M.E. Elzain, D.E. Ellis, D. Guenzburger, Phys. Rev. B 34 (1986) 1430.

[24] B. Lingren, J. Sjöström, J. Phys. F: Metal Phys. 18 (1988) 1563. 